\title{
Cardiorespiratory Coordination after Training and Detraining. A Principal Component Analysis Approach
}

\begin{abstract}
Natàlia Balagué ${ }^{*}$, Jacob González ${ }^{1}$, Casimiro Javierre ${ }^{2}$, Robert Hristovski ${ }^{3}$, Daniel Aragonés ${ }^{4}$, Juan Álamo ${ }^{2}$, Oscar Niño ${ }^{2}$ and Josep L. Ventura ${ }^{2}$

${ }^{1}$ Institut Nacional d'Educació Fisica de Catalunya, Department of Health and Applied Sciences, University of Barcelona, Barcelona, Spain, ${ }^{2}$ Physiological Sciences II, L'Hospitalet de Llobregat, University of Barcelona, Barcelona, Spain, ${ }^{3}$ Faculty of Physical Education, Sport and Health, Ss. Cyril and Methodius University in Skopje, Skopje, Macedonia, ${ }^{4}$ Department of Education, Johannes Gutenberg-Universität Mainz, Mainz, Germany
\end{abstract}

Our purpose was to study the effects of different training modalities and detraining on cardiorespiratory coordination (CRC). Thirty-two young males were randomly assigned to four training groups: aerobic (AT), resistance (RT), aerobic plus resistance (AT + RT), and control (C). They were assessed before training, after training (6 weeks) and after detraining ( 3 weeks) by means of a graded maximal test. A principal component (PC) analysis of selected cardiovascular and cardiorespiratory variables was performed to evaluate $\mathrm{CRC}$. The first $\mathrm{PC}\left(\mathrm{PC}_{1}\right)$ coefficient of congruence in the three conditions (before training, after training and after detraining) was compared between groups. Two PCs were identified in $81 \%$ of participants before the training period. After this period the number of PCs and the projection of the selected variables onto them changed only in the groups subject to a training programme. The $\mathrm{PC}_{1}$ coefficient of congruence was significantly lower in the training groups compared with the $\mathrm{C}$ group $\left[H_{(3, N=32)}=11.28\right.$; $p=0.01]$. In conclusion, training produced changes in CRC, reflected by the change in the number of PCs and the congruence values of $\mathrm{PC}_{1}$. These changes may be more sensitive than the usually explored cardiorespiratory reserve, and they probably precede it.

Keywords: cardiorespiratory exercise testing, complex adaptive systems, principal component analysis, coordinative variables, physiological variables, strength variables, training effects

\section{INTRODUCTION}

Cardiorespiratory exercise testing is commonly used in clinical practice for both functional and diagnostic assessments of all types of populations. It measures a broad range of variables related to cardiorespiratory function with the goal of quantitatively linking metabolic, cardiovascular, and pulmonary responses to exercise (Balady et al., 2010). However, it provides little information about the coordinated activity of these subsystems. Although the study of cardiorespiratory coordination (CRC) is in its infancy and needs better assessment methods (Friedman et al., 2012), there is already some published evidence relating aging and disease with alterations in cardiorespiratory coupling (García et al., 2013; Iatsenko et al., 2013).

As a complex system the human organism acts as an indivisible and integrated whole that cannot be reduced to the quantitative analysis of its subsystem functions (West, 2006). Cardiovascular and cardiorespiratory subsystems are interdependent and interact in a dynamic and nonlinear way, that is, non-proportionally, and they therefore need to be approached through nonlinear models 
(Goushcha et al., 2014), the study of time series, and by using complex systems methodologies (Schulz et al., 2013) These methodologies, focusing on the coordinative aspects of human physiology, must be considered when studying the effects of training on cardiorespiratory function. Furthermore, as our organism tackles every new situation with an existing set of capabilities (Kelso, 1995) and continuously exchanges information with its environment, its behavior is unique and unexpected in the short term (weeks, months) (Hristovski et al., 2010), which is the usual duration of common training programmes.

In order to study the coordination between subsystems involving multiple variables, complex systems approaches propose the detection of coordination variables (the so-called order parameters or collective variables), because they capture the order or coordination of the whole system under study (Haken, 1983). Principal component analysis (PCA) is a common statistical technique that has been used to identify these coordination variables in a wide range of biological research fields including motor control (Newell and Vaillancourt, 2001), brain dynamics (Le Van Quyen, 2003), DNA replication (Elsawy et al., 2005), and protein folding (Maisuradze et al., 2009). PCA reduces the data dimension of coupled systems, extracting the smallest number of components that account for most of the variation in the original multivariate data and summarizing it with little loss of information. Although the PCA applied to kinematic variables has been successfully used to study the effects of motor learning processes (Newell and Vaillancourt, 2001), it has yet to be applied to study training effects on physiological variables. The PCA can be used to identify the degree to which time patterns of physiological responses co-vary, i.e., how much their increases and decreases are statistically synchronized. Two or more cardiorespiratory variables that co-vary in time may show different degree of co-variation: the more they co-vary the more variance they share and the more mutual information they create. This common variance and mutual information makes possible to represent the time patterns of single cardio-respiratory variables using fewer coordination variables (PCs). PCs are extracted in decreasing order of importance so that the first $\mathrm{PC}\left(\mathrm{PC}_{1}\right)$ accounts for as much of the variation as possible, with each successive component accounting for a little less. The number of PCs reflects the dimensionality of the system, such that a decrease in the number of PCs is indicative of greater coordination (fewer dimensions), and vice-versa. The number of PCs changes when the system suffers a qualitative or coordinative reconfiguration. Two aspects of the PCA can be usefully exploited. The first one may be called pragmatic, and consists of making the system under study easier to model by reducing the initial high dimension to significantly lower one (e.g., from six cardio-respiratory variables to one or two PCs). The second one is explanatory and consists of gaining insight in the degree of co-relatedness or its absence among the variables under study. Both aspects may present advantages over other methods for predicting the cardiovascular responses and outcomes.

Two main types of training programmes (aerobic, AT and resistance, RT) have been widely investigated due to their important and different physiological effects (Kenney et al., 2015). Their combination (AT + RT) has recently been recommended for health purposes in a wide variety of populations (Pollock et al., 2000).

Given that cardiorespiratory function cannot be tested solely through quantitative measures, and since there are no studies evaluating the effects of training programmes and detraining on CRC, we aim to investigate the qualitative changes in CRC in healthy young men before and after a period of 6 weeks of different training modalities (AT, RT, and AT $+\mathrm{RT}$ ), and then again 3 weeks after detraining. We specifically hypothesize (1) that training will produce a reduction in the number of PCs, in other words, that it will improve CRC, and (2) that the qualitative information obtained through our approach will complement the quantitative data that are usually obtained.

\section{MATERIALS AND METHODS}

\section{Participants}

Thirty-two healthy, physically active males (age $21.2 \pm 2.4$ years, height $177.1 \pm 6.6 \mathrm{~cm}$, mean body mass $71.0 \pm 5.1 \mathrm{~kg}$ and mean body mass index $22.6 \pm 1.7 \mathrm{~kg} \cdot \mathrm{m}^{-2}$ ), all physical education students with no sport specialization but who engaged in a wide range of aerobic activities at least three times a week, volunteered to participate in the study. After the baseline tests they were randomly assigned to four groups for the 6 weeks of training: aerobic (AT), resistance (RT), aerobic + resistance $(\mathrm{AT}+\mathrm{RT})$, and control (C), without changes in physical activity.

\section{Procedure}

Participants completed a standard medical questionnaire in order to confirm their healthy status and level of physical activity. They also signed an informed consent form. All experimental procedures were approved by the Bio-ethics commission of Barcelona University. After the baseline cardiorespiratory testing and maximal strength and power tests (see below), they followed their assigned specific training programme three times a week:

1. AT group $(n=8)$ : participants pedaled $60 \mathrm{~min}$ at $60 \%$ of their individual maximum workload $\left(60 \% \mathrm{~W}_{\max }\right)$. This workload was increased by $5 \%$ weekly unless the participant was unable to maintain the pace throughout the session. Heart rate was monitored during all the sessions.

2. RT group $(n=8)$ : participants performed a 30-min strength circuit twice (Pollock et al., 2000). Starting weights were $40 \%$ of 1RM for the upper body [i.e., squat, chest press, shoulder press, triceps extension, biceps curl, and pull-down (upper back)] and $60 \%$ for the lower body [quadriceps extension, leg press, leg curls (hamstrings), and calf raise]. Participants were allowed a maximum of 12 repetitions, which included a slow controlled movement ( $2 \mathrm{~s}$ up and $4 \mathrm{~s}$ down). The resting period between exercises was $2 \mathrm{~min}$. Workloads were adjusted weekly, with resistance being increased as needed (typically $5-10 \%$ ) if the participant was able to lift the weight comfortably (i.e., more than 12 repetitions).

3. AT $+\mathrm{RT}$ group $(n=8)$ : participants pedaled at $60 \% \mathrm{~W}_{\max }$ for $30 \mathrm{~min}$ and performed the strength circuit (same as the R group) once. 
4. C group $(n=8)$ : control participants continued with their usual activities, without any special training.

\section{Cardiorespiratory Testing}

The incremental cycling test (Excalibur, Lode, Groningen, Netherlands) started at $0 \mathrm{~W}$ and the workload was increased by $20 \mathrm{~W} / \mathrm{min}$ until participants could not maintain the prescribed cycling frequency of $70 \mathrm{rpm}$ for more than 5 consecutive seconds. During the test, participants breathed through a valve (Hans Rudolph, 2700, Kansas City, MO, USA) and respiratory gas exchange was determined using an automated open-circuit system (Metasys, Brainware, La Valette, France). Oxygen and $\mathrm{CO}_{2}$ content and air flow rate were recorded breath by breath. Before each trial, the system was calibrated with a mixture of $\mathrm{O}_{2}$ and $\mathrm{CO}_{2}$ of known composition $\left(\mathrm{O}_{2} 15 \%, \mathrm{CO}_{2} 5 \%, \mathrm{~N}_{2}\right.$ balanced) (Carburos Metálicos, Barcelona, Spain), as well as with ambient air.

Haemodynamic information was obtained from participants using non-invasive finger cuff technology (Nexfin, BMEYE Amsterdam, Netherlands). The Nexfin device provides continuous blood pressure (BP) monitoring from the resulting pulse pressure waveform, and calculates both systolic and diastolic blood pressure (SBP and DBP). Participants were connected by wrapping an inflatable cuff around the middle phalanx of the finger. The finger artery pulsing is "fixed" to a constant volume by application of an equivalent change in pressure against the blood pressure, resulting in a waveform of the pressure (clamp volume method). The measurement was performed in the non-dominant arm; relaxed, supported by a measurement cable attached by a rubber band. We enabled continuous monitoring finger photoplethysmography, which is useful for assessing acute BP changes (Eckert and Hornskotte, 2002). Electrocardiogram (ECG) was continuously monitored (DMS Systems, DMS-BTT wireless Bluetooth ECG transmitter, and receiver, software DMS Version 4.0, Beijing, China).

All tests were performed in a well-ventilated lab; the room temperature was $23^{\circ} \mathrm{C}$ and the relative humidity $48 \%$, with variations of no more than $1{ }^{\circ} \mathrm{C}$ in temperature and $10 \%$ in relative humidity. The tests were carried out at least $3 \mathrm{~h}$ after a light meal, and participants were instructed not to perform any vigorous physical activity for $72 \mathrm{~h}$ before testing. Participants repeated the tests after6 weeks of training and again after 3 weeks of detraining.

\section{Maximal Strength and Power Testing}

Maximal strength and maximal power of upper and lower limbs, respectively, were measured (Musclelab Power System, Porsgruun, Norway) in each participant. Estimated 1 RM-chest press and 1RM-squat based on submaximal loads was calculated. In the chest press exercise the load started with $25 \mathrm{~kg}$ and continued with $35,45,55,65 \mathrm{~kg}$, etc. In the squat exercise, participants started with $45 \mathrm{~kg}$ and continued with $65,85,105 \mathrm{~kg}$, etc. until they were unable to perform one repetition. Based on these results the maximal 1RM was registered and the force/velocity relationship graph was plotted to determine the maximal power.
All exercise tests were carried out at least $3 \mathrm{~h}$ after a light meal and participants were instructed not to perform any vigorous physical activity for $72 \mathrm{~h}$ before testing. Participants repeated these tests after 6 weeks of training and again after 3 weeks of detraining.

\section{Data Analysis}

The following maximal values of performance and cardiorespiratory variables were registered during the tests: ventilatory threshold in \% of $\mathrm{VO}_{2 \max }$ (by means of the $\mathrm{O}_{2}$ and $\mathrm{CO}_{2}$ ventilatory equivalents method, Reinhard et al., 1979), maximal cycling workload (Wmax), maximal oxygen uptake relative to body weight $\left(\mathrm{VO}_{2} / \mathrm{kg}\right.$.min max), maximal expiratory ventilation per minute $\left(\mathrm{VE}_{\max }\right)$, maximal heart rate $\left(\mathrm{HR}_{\max }\right)$, maximal 1RM-squat, and maximal 1RM-chest. The group means in the different conditions were compared using the non-parametric Friedman ANOVA.

In order to study the CRC in each participant a PCA was performed on the time series of the following selected cardiorespiratory variables: expired fraction of $\mathrm{O}_{2}\left(\mathrm{FeO}_{2}\right)$, expired fraction of $\mathrm{CO}_{2}\left(\mathrm{FeCO}_{2}\right)$, ventilation (VE), systolic blood pressure (SBP), diastolic blood pressure (DBP), and heart rate (HR). Other commonly registered variables in cardiorespiratory testing such as respiratory equivalents, respiratory exchange ratio, oxygen pulse, oxygen consumption, etc. were excluded from the analysis due to their known deterministic mathematical relation with the aforementioned variables. Figure 1 shows a typical example of how the PCA reduces the dimensionality of the six measured cardio-respiratory variables into one or two coordinative variables (PCs). The upper graphs display the time series of the six selected variables, the middle graphs show the degree of unit vector co-linearity of the standardized variables on $\mathrm{PC}_{1}$ and $\mathrm{PC}_{2}$ and the lower graphs the time series of the PCs. As it can be seen there is a reduction in dimensionality from six cardiorespiratory variables (upper graphs) to one or two PCs (lowest graphs). In the middle graphs, unit vectors of the six variables mainly project to, that is, align with, a certain PC. The blue and red lines show the average trends of the two processes (weighted using the least squares method). Correlations $r$ of unit vectors with PCs are given as $r=\cos \alpha$; where $\alpha$ is the angle between the unit vectors and the PCs.

The number of generalizable PCs was determined by the Kaiser-Gutmann criterion, which treats as significant the PCs with eigenvalues $\lambda \geq 1.00$ (Jolliffe, 2002). The optimal parsimony solution of the extracted PCs was obtained by the Varimax orthogonal rotation criterion (Meglen, 1991). In order to compare the structure of the extracted PCs before and after training and after the detraining period we used Tucker's congruence coefficient, which is Salton's cosine similarity measure applied to PCs (Lorenzo-Seva and Ten Berge, 2006).

Since the first PC always contains the largest proportion of the data variance we estimated the congruence coefficients only between the first PCs within each group for the three different conditions (pre, post training, and post detraining condition). The median of the $\mathrm{PC}_{1}$ coefficient of congruence was obtained in each group and condition (before training and after both training and detraining). The null hypothesis of a constant PC congruence 


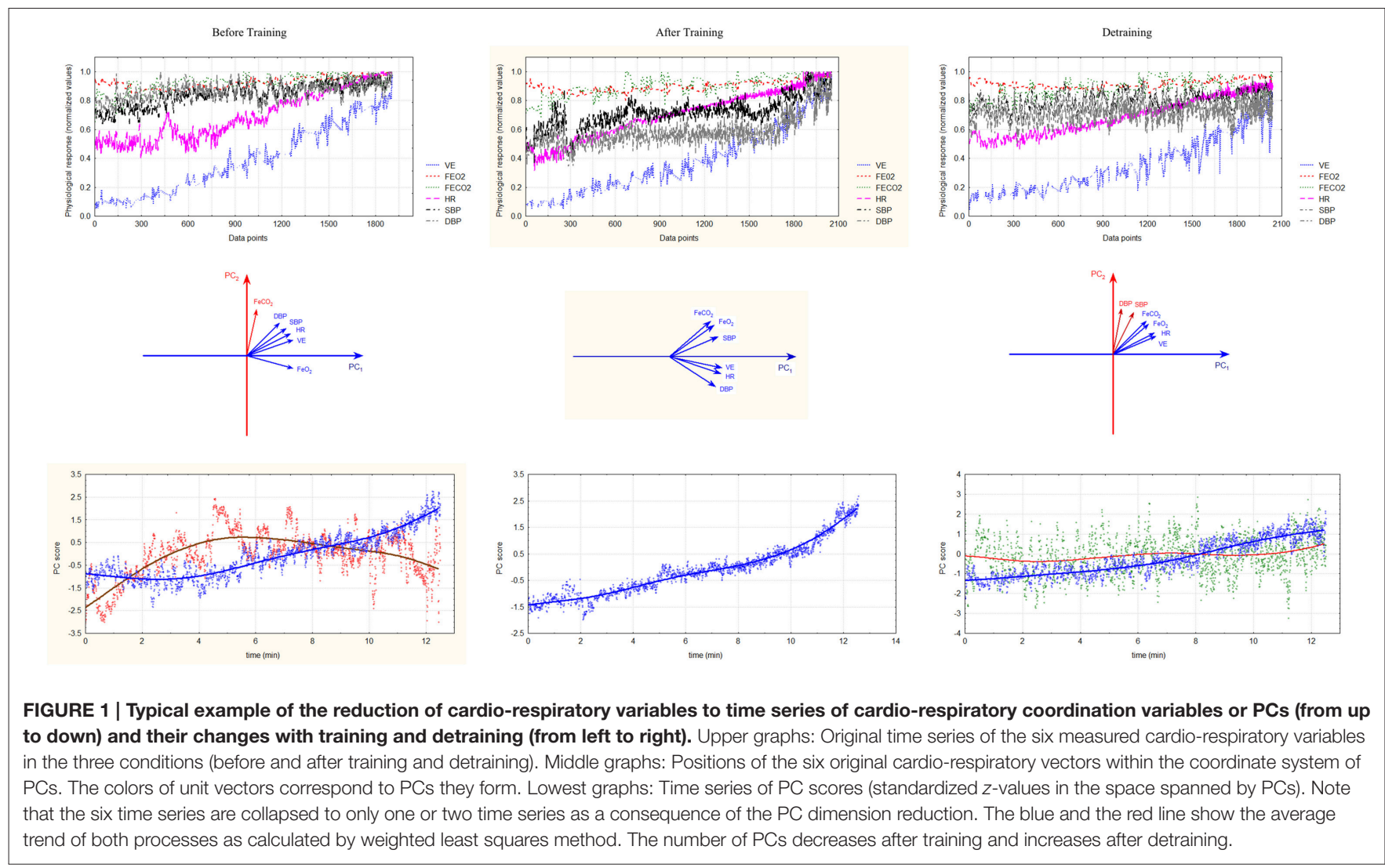

median across the control group and the training groups was tested by means of a non-parametric Kruskal-Wallis ANOVA. Mann-Whitney U matched pairs test analysis was also performed to assess statistically significant differences between each pair of different conditions. Effect sizes (Cohen's $d$ ) were computed to demonstrate the magnitude of standardized differences in medians where effects reached the $p<0.05$ level.

\section{RESULTS}

Table 1 shows the mean and SD of the ventilatory threshold, maximal workload, cardiorespiratory and strength variables registered in the three situations (before training and after both training and detraining) in all groups, not detecting the ANOVA significant differences. Only the AT + RT group showed significant changes in $\mathrm{W}_{\max }$ after the training period $(p=$ 0.02 ). Cohen's $d$-values were 0.7 between the conditions "before training" and "after training" and between "after training" and "after detraining" in this group.

A typical result of the effect of the three conditions (before training, after training, and detraining) on the CRC is shown on Figure 1. In the left column (before training) we see that five variables (VE, $\mathrm{HR}, \mathrm{FeO}_{2}, \mathrm{SBP}$, and $\mathrm{DBP}$ ) show a larger degree of co-linearity with $\mathrm{PC}_{1}$ and that only $\mathrm{FeCO}_{2}$ is dominantly aligned with $\mathrm{PC}_{2}$. Thus, $\mathrm{PC}_{1}$ represents the $\mathrm{CRC}$ and $\mathrm{PC}_{2}$ the idiosyncratic behavior of $\mathrm{FeCO}_{2}$. After training (middle column) the variance of the six cardio-respiratory variables is captured by a sole $\mathrm{PC}_{1}$. This is because the unit vectors of all variables, including $\mathrm{FeCO}_{2}$, are more closely aligned with $\mathrm{PC}_{1}$. Note that it is the shift of the $\mathrm{FeCO}_{2}$ toward the $\mathrm{PC}_{1}$ cluster of variables that enables the formation of this sole $\mathrm{PC}_{1}$. After detraining (right column) the cardio-respiratory variables again form two PCs, pointing to a possible decrease in the coordinative tendencies. This time SBP and DBP are projected in $\mathrm{PC}_{2}$.

Figure 2 shows the percentage of participants having one $\mathrm{PC}$ $\left(\mathrm{PC}_{1}\right)$ or two $\mathrm{PCs}\left(\mathrm{PC}_{1}\right.$ and $\left.\mathrm{PC}_{2}\right)$ in the three conditions in each experimental group. Before training two PCs where found in $81.3 \%$ of participants and a single $\mathrm{PC}$ in $16.6 \%$ of participants. Four variables (VE, $\mathrm{HR}, \mathrm{SBP}, \mathrm{DBP}$ ) plus $\mathrm{FeCO}_{2}$ or, alternatively, $\mathrm{FeO}_{2}$ were always involved in forming $\mathrm{PC}_{1}$, whereas $\mathrm{PC}_{2}$ was formed by a single variable: either $\mathrm{FeCO}_{2}$ or $\mathrm{FeO}_{2}$. The number of PCs was reduced after training in the AT, RT, and AT + RT groups (54\% of participants had two PCs and 45.8 had one PC), returning to initial values after detraining, whereas no changes were observed in the $\mathrm{C}$ group. Table 2 shows the mean and SD of the loading of the selected cardiorespiratory variables onto $\mathrm{PC}_{1}$ in the three conditions inall groups. $\mathrm{PC}_{1}$ was saturated by the loading of $\mathrm{FeO}_{2}, \mathrm{VE}, \mathrm{SBP}, \mathrm{DBP}$, and $\mathrm{HR}$, while $\mathrm{PC}_{2}$ was saturated bythe projection of $\mathrm{FeCO}_{2}$ or, alternatively, by $\mathrm{FeO}_{2}$. The eigenvalues of $\mathrm{PC}_{1}$ increased significantly in $\mathrm{RT}\left[\chi_{(8,2)}^{2}=\right.$ $10.75, p=0.005]$ and AT $+\mathrm{RT}\left[\chi_{(8,2)}^{2}=1.56, p=0.003\right]$ but not in the AT (with large SD) and $\mathrm{C}$ groups (see Table 3 ). The medians of the $\mathrm{PC}_{1}$ coefficient of congruence between the three conditions (before training and after both training and 
TABLE 1 | Means (standard deviations) of ventilatory threshold, maximal workload, and maximal cardiorespiratory and strength variables.

\begin{tabular}{|c|c|c|c|c|c|c|c|c|}
\hline Group & Condition & $\begin{array}{l}\text { VEmax } \\
\left(1 \cdot \min ^{-1}\right)\end{array}$ & $\begin{array}{c}\mathrm{VO}_{2} \max \\
\left(\mathrm{ml} \cdot \mathrm{Kg} \cdot \mathrm{min}^{-1}\right)\end{array}$ & $\begin{array}{c}\text { VTh } \\
\left(\% \mathrm{VO}_{2} \text { max }\right)\end{array}$ & $\begin{array}{c}\text { HRmax } \\
\left(\text { b. } \min ^{-1}\right)\end{array}$ & W max (W) & $\begin{array}{l}\text { Chest press max. } \\
\qquad(\mathbf{k g})\end{array}$ & $\begin{array}{l}\text { Squat max. } \\
\text { (kg) }\end{array}$ \\
\hline \multirow[t]{6}{*}{ AT } & 1 & 125.5 & 51.3 & 40.5 & 185.8 & 301.9 & 62.8 & 167.8 \\
\hline & & (28) & $(9.7)$ & $(2.8)$ & $(10.7)$ & (17.4) & (6.3) & $(29.2)$ \\
\hline & 2 & 149.8 & 60.1 & 47.4 & 184.2 & 328.7 & 68.5 & 166.4 \\
\hline & & (34.8) & $(9.7)$ & (3.2) & (16.9) & (13.3) & $(4.7)$ & $(25.4)$ \\
\hline & 3 & 137.1 & 57.5 & 44.2 & 182.6 & 312.8 & 71.4 & 165.7 \\
\hline & & $(25.1)$ & $(9.1)$ & $(4.3)$ & (15.6) & (16.5) & $(4.7)$ & (30.3) \\
\hline \multirow[t]{6}{*}{$\mathrm{RT}$} & 1 & 119 & 47.3 & 36.8 & 183.9 & 270.2 & 63.3 & 147.5 \\
\hline & & (22.9) & $(6.4)$ & $(1.8)$ & (12.5) & (23.4) & $(5.7)$ & (16.6) \\
\hline & 2 & 124.8 & 49.6 & 41.4 & 177.9 & 273 & 72.5 & 165.6 \\
\hline & & (22.5) & $(7.7)$ & (2.9) & (14.3) & (23.5) & (5.3) & (16.5) \\
\hline & 3 & 117.9 & 50.3 & 36.6 & 177.0 & 267.8 & 72.5 & 151.2 \\
\hline & & $(23.8)$ & $(5.2)$ & $(1.5)$ & (12.9) & (19.6) & $(9.2)$ & $(24.8)$ \\
\hline \multirow[t]{6}{*}{$A T+R T$} & 1 & 117.8 & 45.1 & 40.3 & 175.9 & 273.3 & 71.6 & 156.1 \\
\hline & & $(15.7)$ & (5.3) & (4.3) & (10) & $(12.7)$ & (19.5) & $(24.7)$ \\
\hline & 2 & 123.1 & 49.6 & 39.3 & 171.9 & 302.6 & 76.6 & 158.3 \\
\hline & & (19.6) & (3.8) & (2.4) & $(9.8)$ & (7.5) & (16.3) & $(24.8)$ \\
\hline & 3 & 125.9 & 51.2 & 38.9 & 173.5 & 290. & 81.6 & 158.8 \\
\hline & & (19.8) & $(8.1)$ & 3 & $(12.1)$ & (8.9) & (15.8) & $(24)$ \\
\hline \multirow[t]{6}{*}{ C } & 1 & 125.7 & 51.2 & 37.8 & 184.6 & 280.8 & 67.2 & 167.2 \\
\hline & & $(24.8)$ & $(7.8)$ & $(2.8)$ & $(9.3)$ & (28.8) & (10.3) & $(27.2)$ \\
\hline & 2 & 120.2 & 50.5 & 44.3 & 175.3 & 278.2 & 73.3 & 160 \\
\hline & & $(24.8)$ & (7.4) & (3.5) & (7.7) & $(26.7)$ & (10.3) & (30.3) \\
\hline & 3 & 123.6 & 49.4 & 38.5 & 177.1 & 277.4 & 76.1 & 148.7 \\
\hline & & (25.3) & (6.9) & (2.6) & (10.2) & (32.5) & (10.5) & (34.6) \\
\hline
\end{tabular}

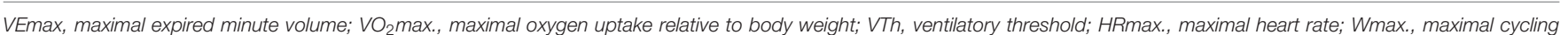
workload; AT, aerobic training; RT, resistance training; $A T+R T$, mixed training; $C$, control; 1, before training; 2, after training; 3, after detraining.

detraining) in the different groups are compared in Figure 3. The size of the $\mathrm{PC}_{1}$ coefficient of congruence was lower in the groups subject to training, as compared with the $\mathrm{C}$ group $\left[H_{(3, N=32)}=\right.$ 11.28; $p=0.01$, with large effect sizes between $\mathrm{C}$ and AT $(d=1.5), \mathrm{C}$ and RT $(d=0.8)$, and C and AT + RT $(d=0.7)$.

\section{DISCUSSION}

The present study was conceived to examine coordinative dimensional changes in cardiorespiratory testing results after a period of training and 3 weeks after detraining. Our hypothesis was based on two assumptions: (a) complex adaptive systems behave as a whole and thus they cannot be evaluated solely through quantitative measurements of isolated subsystem variables, and (b) PCA, providing information about the dimensionality of CRC, is able to capture the qualitative changes produced by training and detraining. Accordingly, our method of assessing CRC was able to provide qualitative information that complements the usually tested quantitative cardiorespiratory reserve and maximal performance values. Our findings indicated that: (a) the selected cardiorespiratory variables $\left(\mathrm{FeO}_{2}, \mathrm{FeCO}_{2}\right.$, VE, SBP, DBP, and HR) obtained through an incremental cardiorespiratory test were reduced in $81.2 \%$ of participants to two dimensions $\left(\mathrm{PC}_{1}\right.$ and $\left.\mathrm{PC}_{2}\right)$, summarizing the integrated $\mathrm{CRC}$ in young trained males before training; (b) only in the training groups was the number of PCs reduced after training, with the initial dimensionality being recovered after detraining; (c) the variables loading onto $\mathrm{PC}_{1}$ remained constant in the $\mathrm{C}$ group but changed in different ways after training and detraining under the different training modalities; (d) the $\mathrm{PC}_{1}$ coefficient of congruence after both training and detraining was significantly lower in the training groups compared with the C group; and (e) the dimensionality reduction in the CRC seemed to precede the quantitative physiological and performance changes that usually follow training and detraining.

Although most of the variables projected onto $\mathrm{PC}_{1}$ were highly correlated (see Table 2) they did not show perfect coupling with the dimension, in other words, none of them totally explained $\mathrm{PC}_{1}$. Only in the training groups did the projected variables and their correlation values change after the training intervention, indicating that a coordinative, nonlinear reconfiguration occurred after training. It is worth noting that the term "nonlinear" used in this paper does not refer to a non-linear fitting (e.g., exponential or logarithmic) of a theoretical curve to the data, but rather to a qualitative change in the data series. Thus, CRC is not rigid, but 


\section{Percentage of participants with $1 \mathrm{PC}$ and $2 \mathrm{Pcs}$}

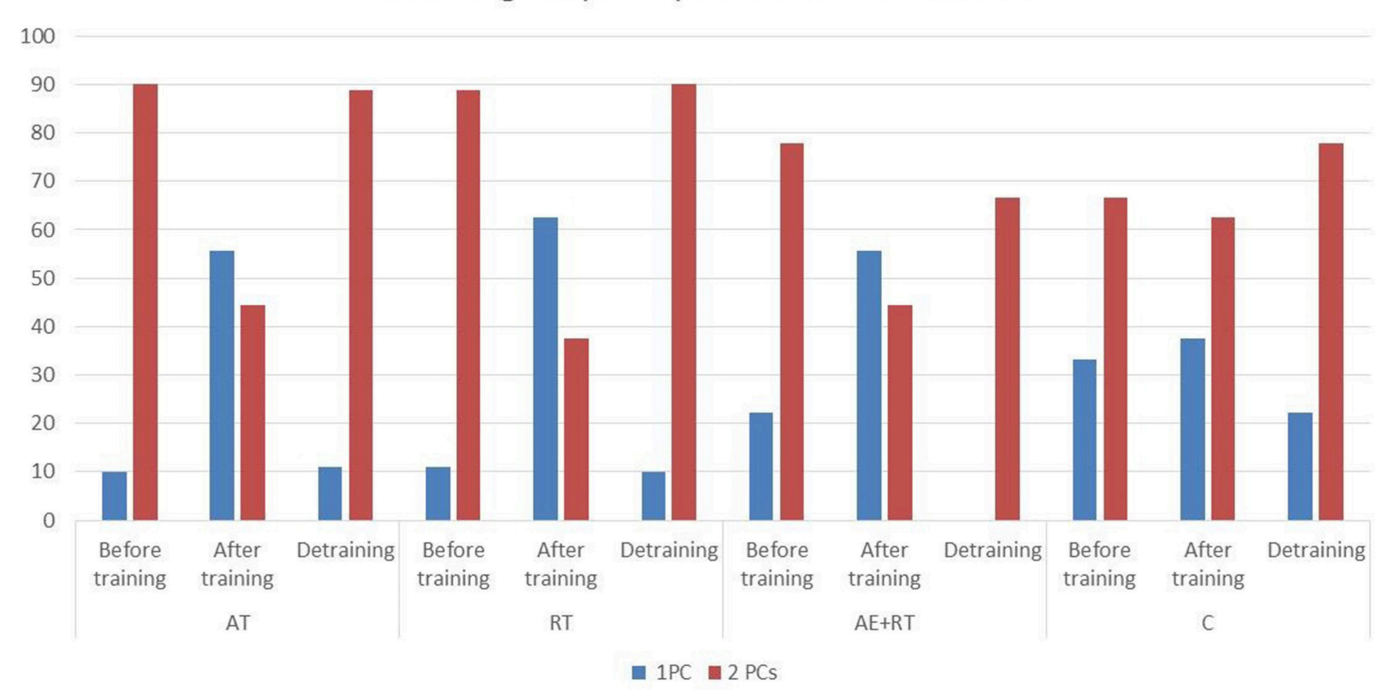

FIGURE 2 | Percentage of participants with one $\mathrm{PC}\left(\mathrm{PC}_{1}\right)$ and two $\mathrm{PCs}\left(\mathrm{PC}_{1}\right.$ and $\left.\mathrm{PC}_{2}\right)$ in the three conditions in all groups. AT, aerobic training; RT, resistance training; AT + RT, mixed training; C, control; 1, before training; 2, after training; 3 , after detraining. In RT group one participant had three PCs before training and after detraining and in AT + RT group one participant had three PCs after detraining.

TABLE 2 | Projection of the selected cardiovascular and cardiorespiratory variables onto $\mathrm{PC}_{1}$ in all participants.

\begin{tabular}{|c|c|c|c|c|c|c|}
\hline \multirow[t]{2}{*}{ Variables } & \multicolumn{2}{|c|}{ Before training } & \multicolumn{2}{|c|}{ After training } & \multicolumn{2}{|c|}{ After detraining } \\
\hline & $M$ & $S D$ & $M$ & $S D$ & $M$ & $S D$ \\
\hline VE & 0.79 & 0.08 & 0.93 & 0.02 & 0.66 & 0.28 \\
\hline $\mathrm{FeO}_{2}$ & 0.52 & 0.10 & 0.63 & 0.14 & 0.47 & 0.25 \\
\hline $\mathrm{FeCO}_{2}$ & 0.64 & 0.15 & 0.77 & 0.04 & 0.57 & 0.29 \\
\hline $\mathrm{HR}$ & 0.91 & 0.04 & 0.93 & 0.03 & 0.79 & 0.21 \\
\hline SBP & 0.82 & 0.03 & 0.87 & 0.03 & 0.78 & 0.19 \\
\hline DBP & 0.75 & 0.09 & 0.80 & 0.04 & 0.76 & 0.19 \\
\hline
\end{tabular}

$V E$, expired minute volume; $\mathrm{FeO}_{2}$, expired fraction of $\mathrm{O}_{2} ; \mathrm{FeCO}_{2}$, expired fraction of $\mathrm{CO}_{2}$ : $H R$, heart rate; SBP, systolic blood pressure; DBP, diastolic blood pressure.

rather flexible and adaptive to changing requirements (Kelso, 1995).

The concept of CRC used here should be distinguished from the concept of "cardiorespiratory coupling," which refers to the adjustment of heart beats at phases of the respiratory cycle (respiratory sinus arrhythmia). CRC assumes a mutual influence of cardiovascular and respiratory oscillations leading to spontaneous coordination.

The current findings reveal that the main coordinative variable $\left(\mathrm{PC}_{1}\right)$ was loaded by all selected variables except $\mathrm{FeO}_{2}$ or, alternatively, $\mathrm{FeCO}_{2}$, which were included in $\mathrm{PC}_{2}$. This means that $\mathrm{FeCO}_{2}$ and $\mathrm{FeO}_{2}$ were less correlated with the remaining variables. In fact, while VE, $\mathrm{HR}, \mathrm{SBP}$, and $\mathrm{DBP}$ values increased with workload during the incremental test, $\mathrm{FeCO}_{2}$ and $\mathrm{FeO}_{2}$ followed a differentiated pattern of behavior. As has been previously described, $\mathrm{FeCO}_{2}$ increases at exercise onset and decreases approaching exhaustion, while $\mathrm{FeO}_{2}$ follows the
TABLE 3 | Eigenvalues of $\mathrm{PC}_{1}$ in the three conditions in all groups.

\begin{tabular}{|c|c|c|c|c|c|c|}
\hline \multirow[t]{2}{*}{ Group } & \multicolumn{2}{|c|}{ Before training } & \multicolumn{2}{|c|}{ After training } & \multicolumn{2}{|c|}{ After detraining } \\
\hline & $M$ & $S D$ & $M$ & $S D$ & $M$ & $S D$ \\
\hline AT & 3.62 & 0.61 & 4.48 & 0.76 & 3.24 & 0.45 \\
\hline RT & 3.51 & 0.46 & 4.12 & 0.53 & 3.13 & 0.13 \\
\hline $\mathrm{AT}+\mathrm{RT} \mathrm{T}^{*}$ & 3.78 & 0.73 & 4.26 & 0.27 & 3.05 & 0.03 \\
\hline C & 3.95 & 0.68 & 4.23 & 0.47 & 4.07 & 0.36 \\
\hline
\end{tabular}

$A T$, aerobic training; $R T$, resistance training; $A T+R T$, mixed training; $C$, control. ${ }^{*} p<0.05$.

contrary pattern (Skinner and McLellan, 1980). The duration of the decreasing $\mathrm{FeCO}_{2}$ and $\mathrm{FeO}_{2}$ phases probably determines whether a new PC $\left(\mathrm{PC}_{2}\right)$ is formed or not. As is known, the decrease in $\mathrm{FeCO} 2$ at the end of an increasing exercise test is a consequence of the hyperventilation produced by the decrease in blood $\mathrm{pH}$. Thus, if such decrease is manifested less in the data series (i.e., it affects fewer data points) for the same or higher workload this means that the ventilation and/or the buffering are more efficient. After 6 weeks of training, most participants (especially those in the AT group) reduced their initial number of PCs (from 2 PCs to 1 PC). This means that the set of studied variables improved their degree of co-variation and all aligned with $\mathrm{PC}_{1}$. A dimension reduction is a hallmark of formation of coordinative structures (Kelso, 1995), and so, the decrease in the number of PCs can be interpreted as an improvement in the efficiency of the CRC. This was probably due to a greater efficiency of gas exchange, as has been shown when studying the synchronization between heartbeat and respiration (Ben-Tal et al., 2012). The possible improved efficiency of gas exchange was not reflected in quantitative changes of the ventilatory threshold obtained through the ventilatory equivalents method. 


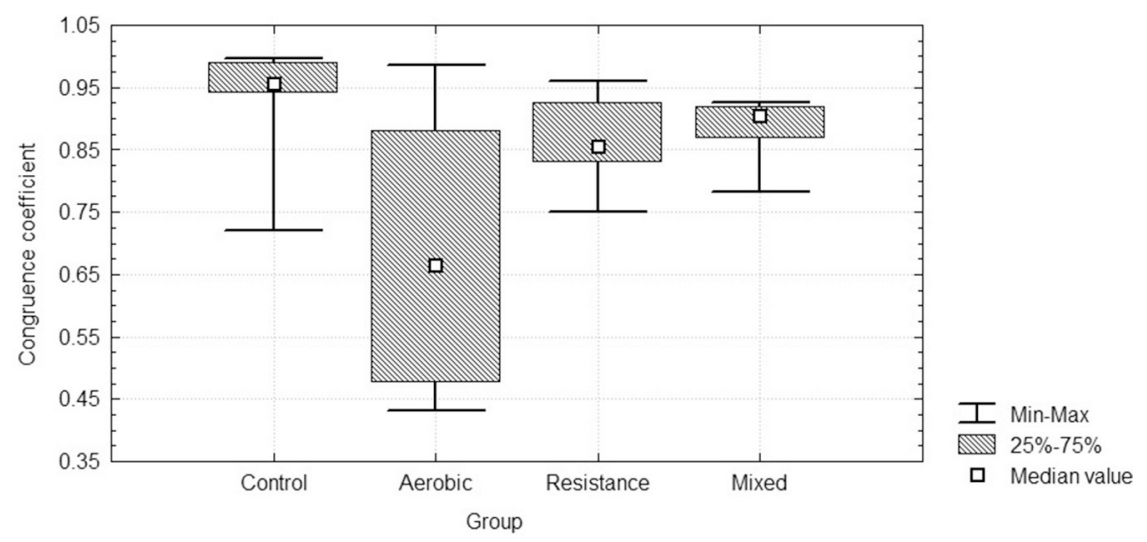

FIGURE 3 | Medians of the $\mathrm{PC}_{1}$ coefficient of congruence between the three conditions (before training and after both training and detraining) in all groups.

However, the relationship between PCA changes and the socalled ventilatory threshold (Reinhard et al., 1979) should be specifically investigated. In fact, the PCA, applied for the first time to the results obtained in cardiorespiratory fitness tests, could help to clarify the controversies around the detection of this threshold.

Although CRC improved after training no significant quantitative increases in cardiorespiratory reserve or performance (ventilatory threshold, $\mathrm{VO}_{2 \max }, \mathrm{W}_{\max }$ and maximal strength and power tests) were observed in the groups under study (see Table 1). Some authors have likewise reported mild or no physiological and performance changes after 6 weeks of aerobic and resistance training in moderately trained participants (Niño et al., 2014) The previous fitness status, combined with the short training period, may explain the negative results obtained here. This means that the CRC assessment may be more sensitive to training and precede the commonly registered quantitative changes. It may also help to discern if, in some cases, the quantitative changes are attributable to training or to other effects (Katch et al., 1982).

The reversibility of the CRC changes with detraining in the AT, $\mathrm{RT}$, and AT + RT groups increased the consistency of the findings. However, these preliminary results need to be confirmed with further research. From a methodological point of view the PCA is a linear dimension reduction technique and consequently it is sensitive only to linear correlational structure within the data. In future research it might be interesting, and even desirable, to apply other statistical techniques for dimension reduction that are more general with this respect, especially its nonlinear generalization such as nonlinear PCA methods (Tenenbaum et al., 2000) or network component analysis (NCA) (Liao et al., 2003). The use of these methods may capture more accurately the curvilinear covariations of physiological signals and hence account for more subtle coordinative tendencies. For example, although the hierarchical PCA is similar to NCA in avoiding orthogonality assumptions about the eigenvectors (PCs), it offers an additional advantage of faithfully reconstructing the network topology when only a partial knowledge is available. The use of these methods in exercise physiology may prove to be fruitful for understanding the intricacies of organic interactions at macroscopic level. From a clinical point of view further research is warranted to clarify (1) if the number of PCs is related to health or training status, (2) if their reduction is linked to better physiological adaptation and performance or health prognosis, (3) the nature of the relationship between CRC and the ventilatory threshold, and (4) the effects of other training programmes such as high-intensity or intervallic training on CRC. As cardiorespiratory exercise testing is applied to a wide range of populations of all ages and training status, future studies should test CRC in athletes of different levels, and also in clinical patients, especially those with cardiorespiratory diseases. Improving the interpretation of cardiorespiratory exercise testing is crucial for healthcare and for wellbeing in general.

The current results show the sensitivity of CRC to training and detraining and highlight the value of incorporating complex systems approaches into the current strategic research framework for sport and exercise medicine (Holtzhausen et al., 2014). However, a longer term study would be required to test the sensitivity of CRC assessments.

\section{AUTHOR CONTRIBUTIONS}

$\mathrm{NB}, \mathrm{CJ}, \mathrm{RH}$, and JV conceived the paper and jointly drafted and reviewed the content; $\mathrm{RH}$ conceived the approach to data analysis; JG, DA, JA, and ON worked on acquisition and analysis of the data. The authors approved the final version and agree to be accountable for all aspect of the work. Conception and design of the work.

\section{FUNDING}

This study has been supported by the Institut Nacional d'Educació Física de Catalunya (INEFC), Generalitat de Catalunya.

\section{ACKNOWLEDGMENTS}

We would like to thank TECNO SPORT for the technical support. 


\section{REFERENCES}

Balady, G. J., Sietsema, K., Myers, J., Coker, L., Fletcher, G. F., Forman, D., et al. (2010). AHA Scientific Statement. Clinician's guide to cardiopulmonary exercise testing in adults. Circulation 122, 191-225. doi: 10.1161/CIR.0b013e3181e52e69

Ben-Tal, A., Shamailov, S. S., and Paton, J. F. R. (2012). Evaluating the physiological significance. J. Physiol. 590, 1989-2008. doi: 10.1113/jphysiol.2011.222422

Eckert, S., and Hornskotte, D. (2002). Comparison of Portapres non-invasive blood pressure measurement in the finger with intra-aortic pressure measurement during incremental bycicle exercise. Blood Press. Monit. 7, 179-183. doi: 10.1097/00126097-200206000-00006

Elsawy, K. M., Hodgson, M. K., and Caves, L. S. D. (2005). The physical determinants of the DNA conformational landscape: an analysis of the potential energy surface of single-strand dinucleotides in the conformational space of duplex DNA. Nucleic Acids Res. 33, 5749-5762. doi: 10.1093/nar/gki888

Friedman, L., Dick, T. E., Jacono, F. J., Loparo, K. A., Yeganeh, A., Fishman, M., et al. (2012). Cardio-ventilatory coupling in young healthy resting subjects. J. Appl. Physiol. 112, 1248-1257. doi: 10.1152/japplphysiol.01424.2010

García, A. Jr, Koschnitzky, J. E., Dashevskiy, T., and Ramirez, J.-M. (2013). Cardiorespiratory coupling in health and disease. Auton. Neurosci. 175, 26-37. doi: 10.1016/j.autneu.2013.02.006

Goushcha, A. O., Hushcha, T. O., Christophorov, L. N., and Goldsby, M. (2014). Self-organization and coherency in biology and medicine. Open J. Biophys. 4, 119-146. doi: 10.4236/ojbiphy.2014.44014

Haken, H. (1983). Synergetics, an Introduction: Nonequilibrium Phase Transitions and Self-Organization in Physics, Chemistry, and Biology. New York, NY: Springer.

Holtzhausen, L. J., van Zyl, G. J., and Nel, M. M. (2014). Developing a strategic research framework for sport and exercise medicine. Br. J. Sports Med. 48, 1120-1126. doi: 10.1136/bjsports-2013-093252

Hristovski, R., Venskaityte, E., Vainoras, A., Balagué, N., and Vázquez, P. (2010). Constraints-controlled metastable dynamics of exercise-induced psychobiological adaptation. Medicina (Kaunas) 46, 447-453.

Iatsenko, D., Bernjak, A., Stankovski, T., Shiogai, Y., Owen-Lynch, P. J., Clarkson, P. B. M., et al. (2013). Evolution of cardiorespiratory interactions with age. Philos. Trans. A Math. Phys. Eng. Sci. 371:20110622. doi: 10.1098/rsta.2011.0622

Jolliffe, I. (2002). Principal Component Analysis. New York, NY: Springer.

Katch, V. L., Sady, S. S., and Freedson, P. (1982). Biological variability in maximum aerobic power. Med. Sci. Sports Exerc. 14, 21-25. doi: 10.1249/00005768198201000-00004

Kelso, J. A. S. (1995). Dynamic Patterns: The Self-Organization of Brain and Behavior. Cambridge, MA: MIT Press Ltd.

Kenney, W. L., Wilmore, J. H., and Costill, D. L. (2015). Physiology of Sport and Exercise, 6th Edn. Champaign, IL: Human Kinetics.

Le Van Quyen, M. (2003). Disentangling the dynamic core: a research program for a neurodynamics at the large-scale. Biol. Res. 36, 67-88. doi: 10.4067/S071697602003000100006

Liao, J. C., Boscolo, R., Yang, Y.-L., Tran, L. M., Sabatti, C., and Roychowdhury, V. P. (2003). Network component analysis: reconstruction of regulatory signals in biological systems. Proc. Natl. Acad. Sci. U.S.A. 100, 15522-15527. doi: $10.1073 /$ pnas. 2136632100

Lorenzo-Seva, U., and Ten Berge, J. M. (2006). Tucker's congruence coefficient as a meaningful index of factor similarity. Methodology (Gott). 2, 57-64. doi: 10.1027/1614-2241.2.2.57

Maisuradze, G. G., Liwo, A., and Scheraga, H. A. (2009). Principal component analysis for protein folding dynamics. J. Mol. Biol. 385, 312-329. doi: 10.1016/j.jmb.2008.10.018

Meglen, R. R. (1991). Examining large databases: a chemometric approach using principal component analysis. J. Chemom. 5, 163-179. doi: 10.1002/cem.1180050305

Newell, K. M., and Vaillancourt, D. E. (2001). Dimensional change in motor learning. Hum. Mov. Sci. 20, 695-715. doi: 10.1016/S0167-9457(01) 00073-2

Niño, O., Balagué, N., Aragonés, D., Blasi, J., Alamo, J. M., Corral, L., et al. (2014). CD34+ circulating progenitor cells after different training programs. Int. J. Sports Med. 36, 292-296. doi: 10.1055/s-0034-1394394

Pollock, M. L., Franklin, B. A., Balady, G. J., Chaitman, B. L., Flep, J. L., Fletcher, B., et al. (2000). AHA science advisory. resistance exercise in individuals with and without cardiovascular disease: benefits, rationale, safety, and prescription: an advisory from the committee on exercise, rehabilitation, and prevention, council on clinical cardiology, Americal Heart Association. Position paper endorsed by the American college of sports medicine. Circulation 101, 828-833. doi: 10.1161/01.CIR.101.7.828

Reinhard, U., Müller, P. H., and Schmülling, R.-M. (1979). Determination of anaerobic threshold by the ventilation equivalent in normal individuals. Respiration 38, 36-42. doi: 10.1159/000194056

Schulz, S., Adochiei, F.-C., Edu, I.-R., Schroeder, L., Costin, H., Bär, K.-J., et al. (2013). Cardiovascular and cardiorespiratory coupling analyses: a review. Philos. Trans. A Math. Phys. Eng. Sci. 371:20120191. doi: 10.1098/rsta.2012.0191

Skinner, J. S., and McLellan, T. M. (1980). The transition from aerobic to anaerobic metabolism. Res. Q. Exerc. Sport 1, 234-248. doi: 10.1080/02701367.1980.10609285

Tenenbaum, J. B., de Silva, V., and Langford, J. C. (2000). A global geometric framework for nonlinear dimensionality reduction. Science 290, 1319-1323. doi: $10.1126 /$ science.290.5500.2319

West, B. J. (2006). Where Medicine Went Wrong. Rediscovering the Path to Complexity. Hackensack, NJ: World Scientific Pub.

Conflict of Interest Statement: The authors declare that the research was conducted in the absence of any commercial or financial relationships that could be construed as a potential conflict of interest.

Copyright (C) 2016 Balagué, González, Javierre, Hristovski, Aragonés, Álamo, Niño and Ventura. This is an open-access article distributed under the terms of the Creative Commons Attribution License (CC BY). The use, distribution or reproduction in other forums is permitted, provided the original author (s) or licensor are credited and that the original publication in this journal is cited, in accordance with accepted academic practice. No use, distribution or reproduction is permitted which does not comply with these terms. 